\title{
Pediatric tracheotomy: indications and decannulation outcomes
}

\author{
Jamie L. Funamura, MD ${ }^{1}$, Blythe Durbin-Johnson, PhD², Travis T. Tollefson, MD, MPH, \\ FACS $^{1}$, Jeanette Harrison, MSN, CCNS ${ }^{1}$, and Craig W. Senders, MD, FACS ${ }^{1}$ \\ ${ }^{1}$ Department of Otolaryngology, UC Davis Medical Center, Sacramento, CA \\ 2Division of Biostatistics, Department of Public Health Sciences, UC Davis School of Medicine, \\ Sacramento, CA
}

\begin{abstract}
Objective-The objective of this study was to determine if there are differences in decannulation rates and duration of cannulation between pediatric patients undergoing tracheotomy for different indications.
\end{abstract}

Study Design—Retrospective chart review.

Methods-Medical records for pediatric patients (age 0-18 years) undergoing tracheotomy between January 1, 2003 and May 31, 2012 were retrospectively reviewed. Patients were assigned an indication for tracheotomy from five categories: neurological, cardiopulmonary, upper airway obstruction, craniofacial anomalies, and maxillofacial/laryngotracheal trauma.

Results-Initial chart review identified 124 patients, 113 for whom complete data was available. Of these patients, the indications for tracheotomy were cardiopulmonary disease in 24 (21.2\%), craniofacial anomalies in 12 (10.6\%), neurological impairment in 44 (38.9\%), traumatic injury in $11(9.7 \%)$, and upper airway obstruction in $22(19.5 \%)$. The time to decannulation was shorter for trauma patients compared to cardiopulmonary $(\mathrm{P}=0.044)$ and neurological patients $(\mathrm{P}=0.001)$. A total of $32(31.9 \%)$ patients were decannulated during the study period, with a higher rate in trauma patients (72.7\%) and a lower rate in those with upper airway obstruction (36.4\%) than would be expected under homogeneity. Of the 32 patients who were decannulated, 11 (30.6\%) were decannulated during the same hospitalization in which the tracheotomy was performed.

Conclusion-This study demonstrates a difference in overall decannulation rates and a shorter time to decannulation in children undergoing tracheotomy for maxillofacial and laryngotracheal trauma compared to cardiopulmonary and neurological indications.

Corresponding author: Jamie L. Funamura, MD, 2521 Stockton Blvd., Suite 7200, Sacramento, CA 95817.

CONFLICT OF INTEREST: None

Level of evidence: 4

Portions of this manuscript were presented at the American Society of Pediatric Otolaryngology Spring Meeting in Arlington, VA on April 25-28, 2013

FINANCIAL DISCLOSURES:

i. The statistical analysis for this project was supported by the National Center for Advancing Translational Sciences, National Institutes of Health, through grant \#UL1 TR000002.

ii. No other financial disclosures applicable 


\section{Keywords}

pediatric tracheotomy; tracheostomy; decannulation; upper airway obstruction

\section{Introduction}

Pediatric tracheotomy today is a relatively common procedure with approximately 5,000 procedures performed a year in the United States for a variety of indications. ${ }^{1}$ The most common indications for pediatric tracheotomy, however, have undergone a substantial shift over the past 40 years. During the 1970s, airway obstruction from acute infection such as laryngotracheobronchitis, epiglottitis, and diphtheria were the most common indications for tracheotomy, representing as many as a third of tracheotomies performed. ${ }^{1-4}$ The widespread practice of vaccinations to cover bacteria such as Haemophilus influenzae type $\mathrm{B}$ and Corynebacterium diptheriae, as well as endotracheal intubation for short-term ventilatory support has decreased the number of tracheotomies performed for acute infectious causes. ${ }^{1,5}$ Furthermore, medical advances have resulted in increased survival of premature neonates and those with complex cardiopulmonary anomalies. These patients may require long-term mechanical ventilation and subsequent tracheotomy. The overall result has been a general trend for patients who require tracheotomy to be younger and more likely to have chronic diseases than was the case forty years ago. ${ }^{1,6-9}$

With the changing clinical indications for tracheotomy, the routine post-tracheotomy course has also undergone a significant evolution. Previously, children undergoing tracheotomy would remain as inpatients until the resolution of the infectious etiology. Subsequent decannulation during that same hospitalization was common. Now, increasing numbers of pediatric patients are requiring long-term tracheotomy to address chronic and congenital diseases. ${ }^{1,3,7}$ The decision point for decannulation has thus shifted to the outpatient setting. This necessitates long-term planning and ongoing communication between patient, family, and provider.

Decannulation is frequently a shared goal due to the functional, psychological, and financial burdens of long-term tracheotomy care. Skilled nursing services are usually required for home tracheotomy care with their attendant costs. The cost of home care for a tracheostomydependent child in the United States was estimated in the early 1990s to be approximately $\$ 110,000$ per year, with home nursing accounting for $60 \%$ of the cost. ${ }^{10}$ Tracheotomy patients are also at higher risk of infection, have delayed speech and language acquisition, and difficulties with socialization. ${ }^{6,10}$ Additionally, it has been shown that both patient and caregiver experience a negative effect on quality of life. ${ }^{11}$ Decannulation is thus sought to both decrease healthcare costs and improve quality of life. Many have therefore recognized the need for algorithms to ensure safe and expedient decannulation. ${ }^{9,12}$

The literature on pediatric tracheotomy currently contains limited objective data on decannulation outcomes. Overall rates of successful decannulation in pediatric patients reported in the recent literature (1990s to the present) range from 35-75\%.3,6,13-18 These studies largely represent the patients undergoing tracheotomy for chronic or congenital diseases. However, specific decannulation rates from study to study are difficult to compare 
due to substantial differences in patient populations and institutional practices. Conclusions on concrete and generalizable predictors of successful decannulation are therefore difficult to extract.

Broadly-defined tracheotomy indications have been studied as predictive factors for decannulation. ${ }^{18}$ In their study, Leung and Berkowitz demonstrated that patients who underwent tracheotomy for tracheobronchial toilet had a significantly shorter cannulation time compared to those with airway obstruction or those requiring prolonged mechanical ventilation. Other authors have grouped pediatric tracheotomy indications according to underlying cause, such as: craniofacial anomalies, upper airway obstruction, traumatic injury, and cardiopulmonary disease or neurological impairment. ${ }^{3,4}$ These classifications may be more helpful in allowing meaningful examination of differences in outcomes between distinct clinical groups. It also may allow for better comparisons of other factors involving post-tracheotomy management such as number and types of additional procedures (e.g. airway evaluations) required prior to decannulation.

In order to further characterize significant determinants of decannulation outcomes in the pediatric population, we reviewed the charts of all pediatric patients undergoing tracheotomy or followed for tracheotomy status at our institution over a 10 -year period. We hypothesized that decannulation rates would be higher, and the time to decannulation shorter, in patients with surgically correctable indications for tracheotomy (maxillofacial trauma, upper airway obstruction, and craniofacial anomalies) compared to patients who underwent tracheotomy for chronic cardiopulmonary disease or neurological impairment. We further anticipated that patients with upper airway obstruction and craniofacial anomalies would undergo more procedures after tracheotomy than patients undergoing tracheotomy for other indications.

\section{Materials and Methods}

The design of this study is a retrospective chart review of all pediatric ( $0-18$ years of age) patients undergoing tracheotomy or followed for tracheotomy status at the UC Davis Medical Center (Sacramento, CA). The study spans a 10-year period (between January 1, 2003 and May 31, 2012, with chart review through December 31, 2012). This study was approved by the University of California Davis Institutional Review Board (UCD IRB 306840-1).

Patient charts were reviewed for the following key variables: date and age of patient at tracheotomy, surgical indication, number and type of procedures performed, duration of cannulation, date and age of successful decannulation, length of follow-up, tracheotomy tube parameters (size, appropriate size for age, capping, speaking valve use) at the time of tracheotomy and/or decannulation, timing of recannulation or repeat tracheotomy, and whether or not decannulation occurred during the original hospitalization in which the tracheotomy was performed. A standardized data extraction form was utilized. Patients in which the date of tracheotomy or indication were not extractable were excluded from the study. A single patient in the craniofacial category was followed after at our institution 2 years after tracheotomy with sufficient clinical information to include in our study for a total 
of 12 years of data. All other follow-up periods were equal or less than the study period of 10 years. Hospitalized tracheotomy patients underwent a protocol of a 24-hour capping trial prior to decannulation. Outpatients underwent direct laryngoscopy and bronchoscopy followed by admission for a 24-hour capping trial and decannulation with continuous pulse oximetry monitoring.

The primary indication for tracheotomy was designated from the following five categories: (1) cardiopulmonary, (2) craniofacial anomalies, (3) neurological impairment, (4) traumatic injury, and (5) upper airway obstruction. A secondary indication was assigned from the same categories when appropriate. Patients with traumatic brain or cervical spine injury were grouped under neurological impairment. The goal was to distinguish the requirement for tracheotomy in these patients with chronic ventilator dependence or secretion management issues from maxillofacial and laryngotracheal injuries with reparable anatomic disruption. Obstructive etiologies were further subdivided into craniofacial and upper airway obstruction categories in order to reflect a skeletal or structural framework issue in the former from a soft tissue obstruction in the latter. The goal of this distinction was to separate these patients by the type and extent of surgical intervention needed, such as distraction osteogenesis.

Each surgical procedure that a patient underwent was designated as a major, minor, or airway evaluation procedure. Major procedures were considered to be surgeries altering the external framework of the airway, such as reduction of facial fractures, distraction osteogenesis, or laryngotracheal reconstructions. Minor procedures included excision of suprastomal granulation tissue and laryngotracheal balloon dilations. Airway evaluations were considered procedures in which direct laryngoscopy and bronchoscopy were performed without any additional intervention.

Statistical analyses were conducted using R, version 3.0.0. ${ }^{19}$ Age at tracheotomy and length of follow-up were compared between groups using a one-way analysis of variance model followed by Tukey post-hoc pairwise comparisons. Lengths of follow-up were log transformed prior to analysis. The distribution of the time to decannulation for each indication and the proportions of patients decannulated at 1 and 2 years were estimated using the Kaplan-Meier method, with confidence intervals estimated using Greenwood's method. ${ }^{20}$ Results were demonstrated as a 1 minus the Kaplan-Meier survival estimate to demonstrate the proportion decannulated (rather than the proportion not yet decannulated).

Time to decannulation was compared among indications using a Cox proportional hazards model, with post-hoc testing for pairwise differences conducted using Tukey contrasts. ${ }^{21}$ Age was considered to be a confounding variable, and therefore a model adjusting for age was proposed. The rates of decannulation during the same hospitalization in which the tracheotomy was performed (for patients who were decannulated), were compared between groups using a chi-square test. The numbers of major procedures, minor procedures, and airway evaluations were compared between indications using Kruskal-Wallis tests, followup by pairwise comparisons using Wilcoxon rank-sum tests with Bonferroni-corrected pvalues. 


\section{Results}

One hundred and twenty-four patients met inclusion criteria and their medical records reviewed. After patients with insufficient clinical information were excluded, data from a total of 113 patients were analyzed. Primary indications for tracheotomy were identified along with secondary indications if appropriate. The individual diagnoses categorized as cardiopulmonary, craniofacial, neurological impairment, trauma, or upper airway obstruction are listed in Table 1. Of the 113 patients, 24 (21.2\%) had cardiopulmonary disease, 12 (10.6\%) had craniofacial anomalies, 44 (38.9\%) had neurological impairment, 11 $(9.7 \%)$ had traumatic injury, and $22(19.5 \%)$ had an upper airway obstruction (Table 2). Secondary indications were also identified, and while these numbers were insufficient for data analysis, there is a general trend for significant proportions of patients to have multiple indications for tracheotomy in the craniofacial, cardiopulmonary, and upper airway obstruction categories.

The Kaplan-Meier estimates of time to decannulation for each indication are summarized in Table 3 and Figure 1. The time to decannulation is shorter trauma patients compared to other indications. This conclusion was reached based on the Cox proportional hazards model in which the hazard, or likelihood, of decannulation is higher $(\mathrm{P}=0.002$ for comparison of trauma with cardiopulmonary, $\mathrm{P}<0.001$ for all other groups). When adjusting for age in the Cox proportional hazards model, the time to decannulation was marginally significantly higher in patients with trauma injury than cardiopulmonary disease $(\mathrm{P}=0.044)$ and significantly higher in trauma patients than in neurological patients $(\mathrm{P}=0.001)$.

The mean age of tracheotomy in our study was 5.2 years (Table 2). The age at tracheotomy was significantly older in trauma patients than in cardiopulmonary $(\mathrm{P}=0.003)$, craniofacial $(\mathrm{P}<0.001)$, and upper airway obstruction patients $(\mathrm{P}<0.001)$. The age at tracheotomy is also significantly older in neurological patients compared to craniofacial patients $(\mathrm{P}<0.001)$ and those with upper airway obstruction $(\mathrm{P}=0.003)$. Figure 2 shows boxplots of age at tracheotomy by indication, and Table 4 demonstrates the comparison of mean age and associated $\mathrm{P}$ values by indication.

The mean length of follow-up by the service performing tracheotomy was significantly shorter in trauma patients than craniofacial $(\mathrm{P}=0.010)$, neurological $(\mathrm{P}=0.040)$, and upper airway obstruction patients $(\mathrm{P}=0.004)$; 0.5 years compared to $3.9,2.8$, and 3.3 years. This is also the case for length of follow-up at our institution for the same groups $(\mathrm{P}=0.046, \mathrm{P}=$ $0.030, \mathrm{P}=0.021$ ); 1 year versus $3.9,3.3$, and 3.5 years. However, this is largely due to the shorter time to decannulation in the patients with traumatic injury.

The rate of decannulation during the same hospitalization in which the tracheotomy was performed differed significantly between indications $(P=0.018)$, with examination of adjusted residuals suggesting a higher rate in trauma patients (72.7\%) and a lower rate in upper airway obstruction patients (36.4\%) than would be expected under homogeneity. A total of 32 of the 113 patients were decannulated during the study period (31.9\%). Of the 32 patients who were decannulated, $11(30.6 \%)$ were decannulated during the same hospitalization in which the tracheotomy was performed. Outcomes other than 
decannulation were as follows: 23 (20.3\%) died of causes unrelated to tracheotomy, 23 (20.3\%) were transferred to other institutions or otherwise lost to follow-up, and 35 (31.0\%) were followed as outpatients at the end of the study period.

The number of procedures each patient underwent during the course of the study period was documented and analyzed for differences by indication. A total of 21 major procedures, 55 minor procedures, and 95 airway evaluations were performed during the study period. The number of major procedures was significantly lower in neurological patients $(0.1 \pm 0.4$ procedures) than in patients with upper airway obstructions $(0.4 \pm 0.6, \mathrm{P}=0.033)$. The number of minor procedures was significantly higher in patients with upper airway obstruction $(1.5 \pm 3.1)$ than in cardiopulmonary patients $(0.2 \pm 1.0, \mathrm{P}=0.045)$. The number of airway evaluations was significantly higher in upper airway obstruction patients (3.1 \pm 4.9$)$ than in cardiopulmonary patients $(0.3 \pm 1.2, \mathrm{P}=0.001)$, neurological patients $(0.3 \pm 0.8, \mathrm{P}=$ $0.001)$, or trauma patients $(0, \mathrm{P}=0.011)$.

\section{Discussion}

The results of our study are consistent with and expand upon current literature on decannulation in pediatric patients. Similar to other studies focusing on pediatric tracheostomy outcomes, the indications for the tracheotomy in the majority of patients are primarily those of congenital and chronic conditions. ${ }^{1,3,6-9}$ In our study, congenital and/or chronic diseases as indication for tracheotomy constituted $90 \%$ of patients; acute trauma represented $10 \%$ of patients. Secondary indications were also noted in a substantial number of cardiopulmonary, craniofacial, and upper airway obstruction patients and suggest a possible need to study decannulation outcomes in which there are multiple indications for tracheotomy.

As discussed previously, the reported rates of overall decannulation vary significantly between studies, from 35-75\%.3,6,13-18 Our own reported percentage of decannulation is on the lower end of this spectrum at $31.9 \%$. This is likely due to patient population characteristics. In our study, relatively few patients underwent tracheotomy for trauma (the group most likely to be decannulated), while approximately $60 \%$ of patients required tracheotomy for chronic neurological or cardiopulmonary disease. This is compared to two other studies in which only $30 \%$ of patients underwent tracheotomy due to requirements for chronic ventilatory support. The decannulation rates in these studies were $64 \%$ and $75 \%$ respectively. ${ }^{13,17}$ Additionally, our study period coincides with the beginning of our distraction osteogenesis program for infants with tongue-based airway obstruction (e.g. micrognathia). During the study period, infants with mild-to-moderate micrognathia amenable to distraction were able to avoid tracheotomy, selecting for more severe airwayimpairing craniofacial anomalies in this study.

Both the large proportion of patients requiring long-term ventilatory support and presence of a distraction osteogenesis program at our institution highlight the inherent limitations of this single-institution study. Other patient population characteristics may have significantly impacted our decannulation outcomes. For the majority of the study period, our hospital was the only level 1 trauma center and pediatric cardiac intensive care unit in the area, resulting 
in large numbers of closed head and spinal cord injuries and children with cardiac anomalies requiring long-term ventilatory support. Many children are transferred back to local hospitals and rehabilitation centers that can manage their long-term care without further follow-up at our institution. This presumably affects both our decannulation outcomes and ability to track such patients.

Patients with traumatic injury or neurologic impairment were older than the cardiopulmonary, craniofacial, and upper airway obstruction patients. This age difference might be expected as upper airway obstruction and craniofacial anomalies tend to affect infants and toddlers while older children are more likely to be exposed to traumatic injury. All craniofacial patients, for example, were less than one year of age. Because the trauma and neurological impairment groups differed significantly in age from the other groups studied, age-adjusted decannulated rates were examined. We were then able to demonstrate that trauma patients were more likely to be decannulated at 1 and 2 years after tracheotomy than cardiopulmonary and neurological patients, independent of age.

Only the traumatic and neurological injury patients were decannulated during the original hospitalization in which they underwent tracheotomy. This would suggest that early family involvement in tracheotomy care is certainly warranted for children undergoing tracheotomy for other indications. In general, families and medical/surgical teams should prepare for home tracheotomy care at discharge.

Our own mortality rate of $20 \%$ in pediatric tracheotomy patients is in the upper range of other recently published reports demonstrating a 13.3\%-19\% mortality. In these reports, deaths were almost exclusively due to causes other than tracheotomy, and in our own review there were no tracheotomy-related deaths. $.37,13,15,17$

We expected to find significant differences in the number of airway evaluations as well as major and minor procedures between the upper airway obstruction and craniofacial patients over other categories. Patients with craniofacial anomalies did not demonstrate significant differences in numbers of procedures from other groups. However, patients with upper airway obstruction major and minor procedures as well as airway evaluations than groups with non-obstructive indications for tracheotomy. This would be expected given the nature of these obstructive conditions being amenable to surgical correction in many cases.

In this study we were able to demonstrate a difference in overall decannulation percentage in trauma patients compared to children undergoing tracheotomy for either cardiopulmonary or neurological indications, independent of age. The significantly shorter time to decannulation for trauma patients might be expected with self-limited conditions. A potential application of this information is to work towards developing indication-specific decannulation protocols in which patients who are more likely to be decannulated (such as those with traumatic injuries) can be addressed with more aggressive protocols. Other patients may warrant less aggressive protocols. For example, upper airway obstruction patients typically underwent $3-$ 4 minor procedures or airway evaluations during the course of our study. Despite this, the decannulation rate in this group was not significantly lower. This may suggest that upper 
airway obstruction patients may require less frequent airway evaluations depending on their likelihood of decannulation at successive time intervals.

Our goal moving forward is to be able to identify other factors predictive of successful decannulation in pediatric patients with a prospective study. It is our hope that certain clinical indicators such as tracheotomy tube size for age/weight, ability to use a speaking valve, presence of adequate swallow, or "cough-to-clear" can be identified. Ideally, these clinical predictive factors could be used in determining appropriateness of additional airway evaluations, minimizing the frequency of procedures while taking advantage of a higher likelihood of decannulation. This would ideally merge the use of predictive clinical factors with the likelihood of decannulation based on clinical indication in an indication-specific decannulation algorithm. Both safe and timely decannulation in pediatric tracheotomy patients could thus be facilitated.

\section{Conclusion}

This study demonstrates higher decannulation rates and a shorter time to decannulation in children undergoing tracheotomy for maxillofacial and laryngotracheal trauma compared to cardiopulmonary and neurological indications. Further investigations for clinical predictors of successful decannulation are warranted to facilitate safe and timely decannulation while minimizing the number of invasive procedures in pediatric tracheotomy patients.

\section{Acknowledgments}

The statistical analysis for this project was supported by the National Center for Advancing Translational Sciences, National Institutes of Health, through grant \#UL1 TR000002.

\section{References}

1. Lewis CW, Carron JD, Perkins JA, Sie KC, Feudtner C. Tracheotomy in pediatric patients: a national perspective. Arch Otolaryngol Head Neck Surg. 2003; 129:523-529. [PubMed: 12759264]

2. Hadfield PJ, Lloyd-Faulconbridge RV, Almeyda J, Albert DM, Bailey CM. The changing indications for paediatric tracheostomy. Int J Pediatr Otorhinolaryngol. 2003; 67:7. [PubMed: 12560142]

3. Carron JD, Derkay CS, Strope GL, Nosonchuk JE, Darrow DH. Pediatric tracheotomies: changing indications and outcomes. Laryngoscope. 2000; 110:1099-1104. [PubMed: 10892677]

4. Line WS Jr, Hawkins DB, Kahlstrom EJ, MacLaughlin EF, Ensley JL. Tracheotomy in infants and young children: the changing perspective 1970-1985. Laryngoscope. 1986; 96:510-515. [PubMed: 3702566]

5. Dursun O, Ozel D. Early and long-term outcome after tracheostomy in children. Pediatr Int. 2001; 53:202-206. [PubMed: 20667029]

6. Zenk J, Fyrmpas G, Zimmermann T, Koch M, Constantinidis J, Iro H. Tracheostomy in young patients: indications and long-term outcome. Eur Arch Otorhinolaryngol. 2009; 266:705-711. [PubMed: 18766359]

7. Sidman JD, Jaguan A, Couser RJ. Tracheotomy and decannulation rates in a level 3 neonatal intensve care unit: a 12-year study. Laryngoscope. 2006; 116:136-139. [PubMed: 16481826]

8. Lawrason A, Kavanagh K. Pediatric tracheotomy: are the indications changing? Int J Pediatr Otorhinolaryngol. 2013; 77:922-925. [PubMed: 23537927]

9. Kraft S, Patel S, Sykes K, Nicklaus P, Gratny L, Wei JL. Practice patterns after tracheotomy in infants younger than 2 years. Arch Otolaryngol Head Neck Surg. 2011; 137:670-674. [PubMed: 21502466] 
10. Graf JM. Early decannulation of the pediatric patient from tracheostomy to noninvasive positive pressure ventilation: teamwork and patience. Pediatr Crit Care Med. 2010; 11:146-147. [PubMed: 20051794]

11. Hopkins C, Whetstone S, Foster T, Blaney S, Morrison G. The impact of paediatric tracheostomy on both patient and parent. Int J Pediatr Otorhinolaryngol. 2009; 73:15-20. [PubMed: 19019462]

12. Kubba H, Cooke J, Hartley B. Can we develop a protocol for safe decannulation of tracheostomies in children less than 18 months old? Int J Pedatr Otorhinolaryngol. 2004; 68:935-937.

13. Mahadevan M, Barber C, Salkeld L, Douglas G, Mills N. Pediatric tracheotomy: 17 year review. Int J Pediatr Otorhinolaryngol. 2007; 71:1829-1835. [PubMed: 17953995]

14. French LC, Wootten CT, Thomas RG, Neblett WW 3rd, Werkhaven JA, Cofer SA. Tracheotomy in the preschool population: indication and outcomes. Otolaryngol Head Neck Surg. 2007 Aug. 137:280-283. [PubMed: 17666256]

15. Ozmen S, Ozmen OA, Unal OF. Pediatric tracheotomies: a 37-year experience in 282 children. Int J Pediatr Otorhinolaryngol. 2009; 73:959-961. [PubMed: 19395057]

16. de Trey L, Niedermann E, Ghelfi D, Gerber A, Gysin C. Pediatric tracheotomy: a 30-year experience. J Pediatr Surg. 2013; 48:1470-1475. [PubMed: 23895956]

17. Tantinikorn W, Alper CM, Bluestone CD, Casselbrant ML. Outcome in pediatric tracheotomy. Am J Otolaryngol. 2003; 24:131-137. [PubMed: 12761697]

18. Leung R, Berkowitz RG. Decannulation and outcome following pediatric tracheostomy. Ann Otol Rhinol Laryngol. 2005; 114:743-748. [PubMed: 16285263]

19. R Core Team. R: A language and environment for statistical computing. R Foundation for Statistical Computing; Vienna, Austria: 2013. URL <http://www.R-project.org/>

20. Greenwood, M, Jr. Reports of Public Health and Related Subjects. London, UK: HMSO; 1926. The national duration of cancer; p. 33

21. Kramer CY. Extension of multiple range tests to group means with unequal numbers of replications. Biometrics. 1956:307-310. 


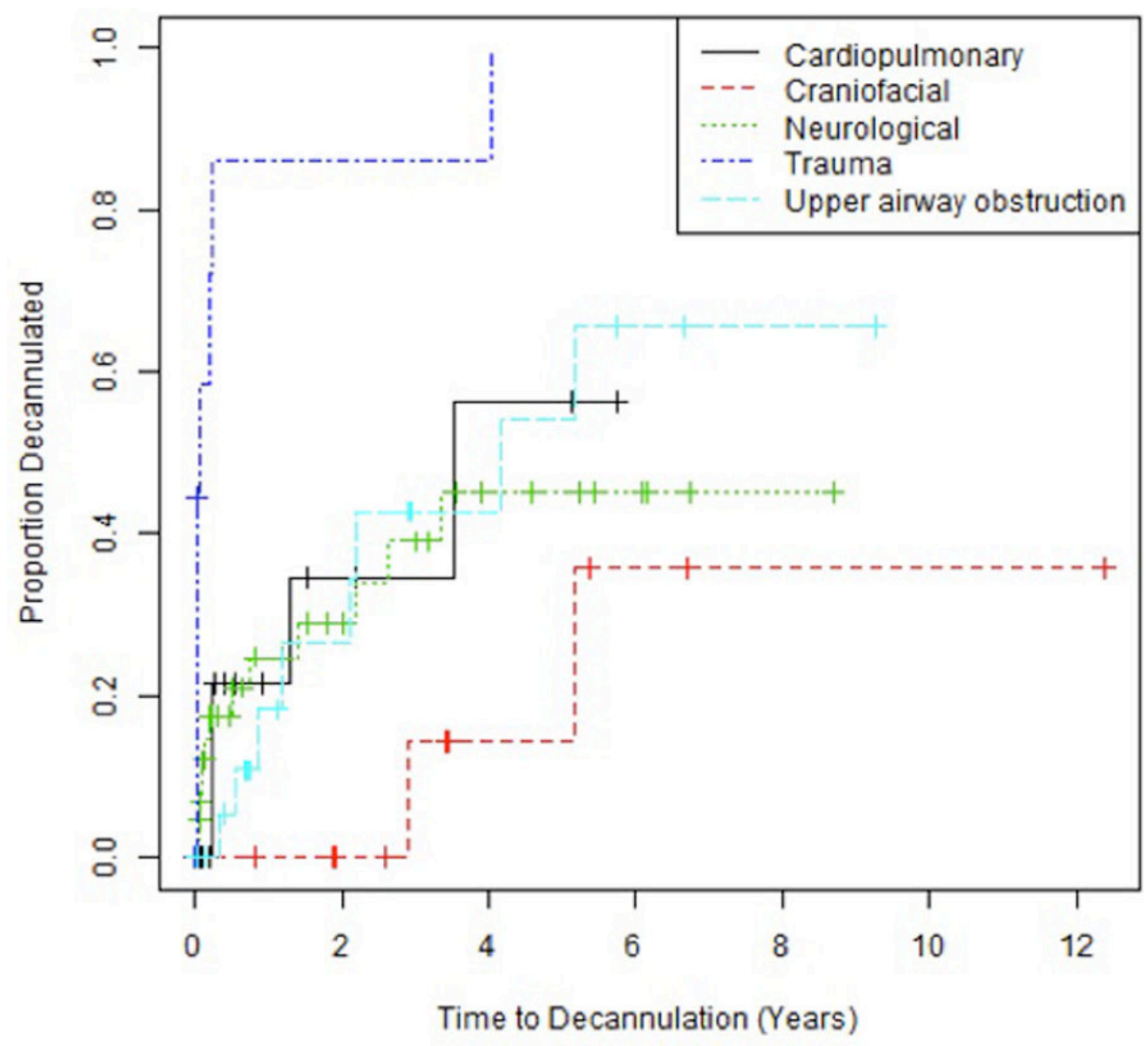

Figure 1.

Kaplan-Meier curves demonstrating time to decannulation, which is shorter for trauma patients compared to other indications $(\mathrm{P}=0.002$ for comparison of trauma with cardiopulmonary, $\mathrm{P}<0.001$ for all other groups). 


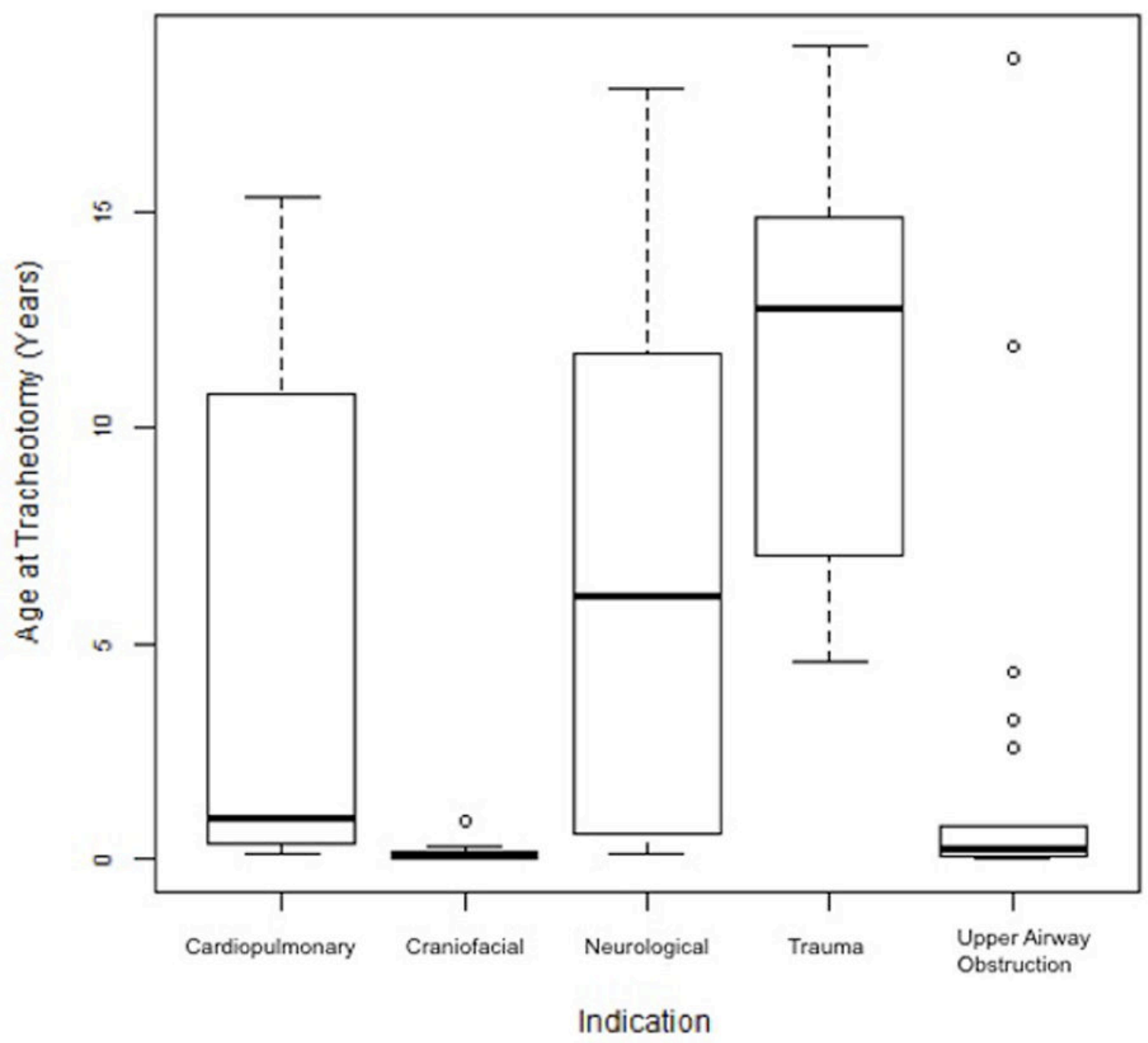

Figure 2.

Boxplots of age at time of tracheotomy by indication. The age at tracheotomy was significantly older in trauma patients than in cardiopulmonary $(\mathrm{P}=0.003)$, craniofacial $(\mathrm{P}<$ $0.001)$, and upper airway obstruction patients $(\mathrm{P}<0.001)$. 


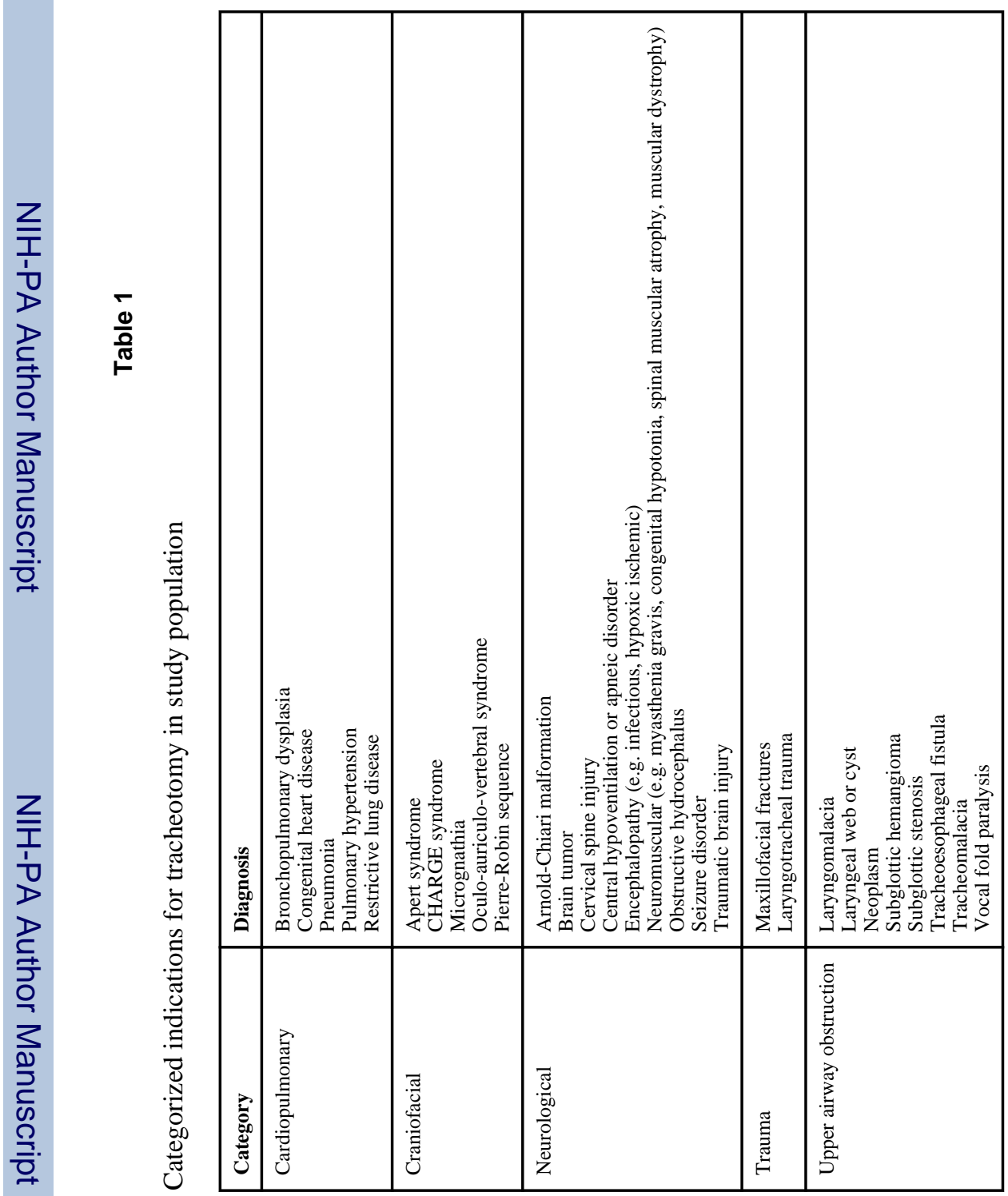

Laryngoscope. Author manuscript; available in PMC 2015 August 01. 


\begin{tabular}{|c|c|c|c|c|c|c|c|c|c|c|c|c|c|c|c|c|c|c|c|}
\hline 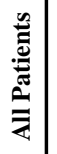 & $\stackrel{\Xi}{=}$ & 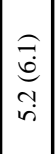 & 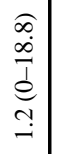 & 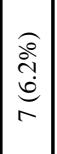 & 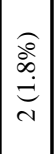 & $\mid \begin{array}{l}0 \\
\stackrel{9}{2} \\
\stackrel{9}{9} \\
=\end{array}$ & 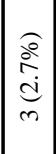 & 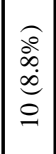 & 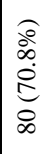 & 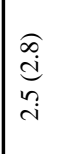 & 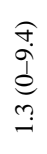 & $\begin{array}{l}\hat{\widehat{d}} \\
\hat{d} \\
\hat{i}\end{array}$ & $\begin{array}{l}6 \\
o \\
e \\
\stackrel{1}{-} \\
\end{array}$ & $\begin{array}{l}\tilde{n} \\
\stackrel{e}{0} \\
\tilde{o} \\
0\end{array}$ & $\begin{array}{c}\widehat{T} \\
\stackrel{1}{0} \\
0\end{array}$ & 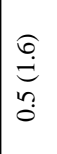 & $\begin{array}{l}\frac{f}{1} \\
\frac{1}{0}\end{array}$ & $\begin{array}{l}0 \\
\stackrel{d}{d} \\
\infty \\
\stackrel{0}{0}\end{array}$ & $\begin{array}{l}\text { E } \\
\text { d } \\
0\end{array}$ \\
\hline 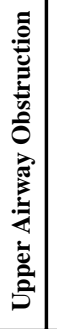 & $\tilde{\pi}$ & 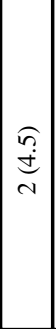 & $\begin{array}{c}n \\
n \\
\infty \\
1 \\
e \\
\tilde{o} \\
0\end{array}$ & 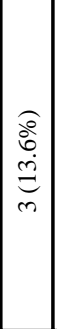 & 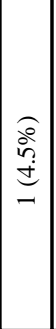 & 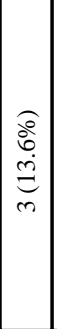 & 0 & 0 & 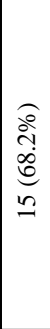 & $\mid \begin{array}{l}\hat{\mathcal{d}} \\
\stackrel{d}{\tilde{d}}\end{array}$ & $\begin{array}{l}\text { f. } \\
\hat{a} \\
\hat{d} \\
n \\
i\end{array}$ & \begin{tabular}{|l}
$\widehat{\hat{n}}$ \\
$n$ \\
$\tilde{n}$
\end{tabular} & 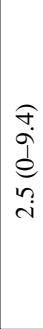 & $\begin{array}{l}\text { U. } \\
\text { é } \\
\dot{0} \\
0\end{array}$ & $\begin{array}{l}\hat{T} \\
\stackrel{1}{0} \\
0\end{array}$ & $\mid \begin{array}{l}\overrightarrow{0} \\
\bar{n} \\
n \\
n\end{array}$ & $\begin{array}{l}\frac{f}{1} \\
\stackrel{d}{0}\end{array}$ & $\begin{array}{l}\stackrel{u}{\sigma} \\
\underset{+}{\dot{\theta}} \\
\vec{r}\end{array}$ & $\stackrel{E}{e}$ \\
\hline 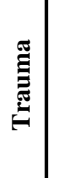 & $=$ & $\mid \begin{array}{c}= \\
\vec{b} \\
n \\
= \\
=\end{array}$ & 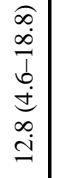 & 0 & 0 & $\begin{array}{c}0 \\
\stackrel{0}{1} \\
\infty \\
\infty \\
\sim \\
\sim\end{array} \mid$ & 0 & 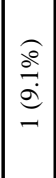 & 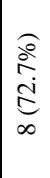 & $\mid \begin{array}{l}0 \\
\hat{e} \\
\dot{e} \\
n \\
0\end{array}$ & 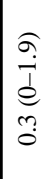 & 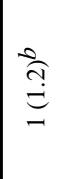 & $\begin{array}{l}\tilde{n} \\
\tilde{o} \\
e \\
\vdots \\
0 \\
0\end{array}$ & 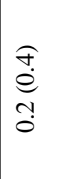 & $\begin{array}{c}\widehat{\bar{d}} \\
0 \\
0\end{array}$ & $\hat{\mathfrak{a}}$ & $\begin{array}{l}\hat{0} \\
0 \\
0 \\
0\end{array}$ & $\stackrel{\varrho}{o}$ & $\begin{array}{l}0 \\
0 \\
0 \\
0\end{array}$ \\
\hline 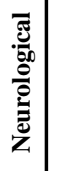 & F & $\begin{array}{c}\widehat{\sigma} \\
\stackrel{6}{r}\end{array}$ & $\begin{array}{c}\sigma \\
\stackrel{\sigma}{\sigma} \\
\frac{1}{e} \\
\overline{0}\end{array}$ & $\mid \begin{array}{c}0 \\
\frac{0}{0} \\
\frac{9}{\sigma} \\
\sigma\end{array}$ & 0 & 0 & 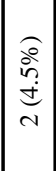 & 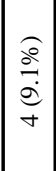 & 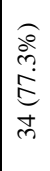 & $\mid \begin{array}{c}\widehat{O} \\
\infty \\
i \\
i\end{array}$ & $\mid \begin{array}{c}\hat{a} \\
\hat{a} \\
\hat{d} \\
n \\
\end{array}$ & 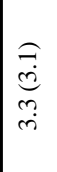 & $\begin{array}{l}\sigma \\
\stackrel{0}{i} \\
\frac{1}{e} \\
i \\
i\end{array}$ & $\begin{array}{l}\stackrel{f}{\stackrel{e}{e}} \\
\stackrel{0}{0}\end{array}$ & $\begin{array}{c}\hat{T} \\
\stackrel{d}{0} \\
0\end{array}$ & 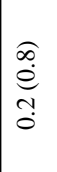 & $\frac{\pi}{\mathfrak{s}}$ & 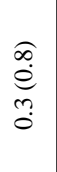 & $\begin{array}{l}f \\
\text { fo } \\
0\end{array}$ \\
\hline 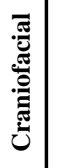 & $\simeq$ & 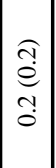 & $\begin{array}{c}\sigma \\
\hat{0} \\
0 \\
0 \\
0\end{array}$ & 0 & 0 & $\begin{array}{l}0 \\
0 \\
d \\
d \\
m\end{array}$ & 0 & 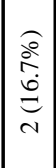 & \begin{tabular}{|l}
0 \\
0 \\
$\infty$ \\
0 \\
0 \\
$r$
\end{tabular} & \begin{tabular}{|l}
$\hat{0}$ \\
ले \\
$\dot{m}$
\end{tabular} & $\begin{array}{l}\hat{a} \\
\hat{d} \\
\dot{j} \\
\dot{r}\end{array}$ & $\begin{array}{l}\hat{\mathfrak{n}} \\
\text { ले }\end{array}$ & $\begin{array}{l}\hat{a} \\
e \\
\vec{j} \\
\dot{r}\end{array}$ & $\begin{array}{l}\hat{e} \\
e \\
\stackrel{0}{0} \\
\dot{0}\end{array}$ & $\begin{array}{c}\hat{T} \\
\stackrel{\jmath}{0} \\
0\end{array}$ & $\begin{array}{l}\hat{\sigma} \\
\hat{e} \\
\hat{o}\end{array}$ & $\begin{array}{c}\hat{\jmath} \\
\varrho \\
0\end{array}$ & $\begin{array}{l}\widehat{C} \\
\stackrel{\Xi}{0} \\
\stackrel{0}{0}\end{array}$ & $\hat{\widehat{\rho}}$ \\
\hline 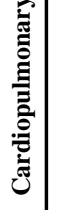 & $\stackrel{d}{d}$ & 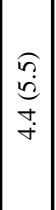 & $\begin{array}{l}\hat{\sigma} \\
\stackrel{0}{1} \\
\frac{1}{0} \\
\stackrel{0}{0}\end{array}$ & 0 & 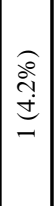 & 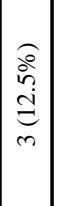 & 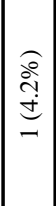 & 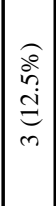 & $\begin{array}{l}0 \\
0 \\
0 \\
0 \\
0 \\
0\end{array}$ & $\mid \begin{array}{c}\widehat{\vec{d}} \\
\stackrel{d}{-}\end{array}$ & 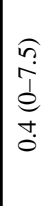 & $\begin{array}{l}\stackrel{\hat{n}}{d} \\
\underset{i}{d}\end{array}$ & $\begin{array}{l}\hat{n} \\
\hat{j} \\
\hat{s} \\
n \\
0\end{array}$ & 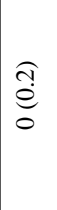 & $\mid \begin{array}{l}\hat{\overline{1}} \\
0 \\
0\end{array}$ & $\begin{array}{l}\widehat{\Xi} \\
\tilde{o} \\
0\end{array}$ & $\frac{n}{0}$ & 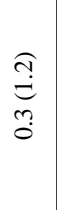 & $\begin{array}{l}0 \\
0 \\
0 \\
0\end{array}$ \\
\hline
\end{tabular}

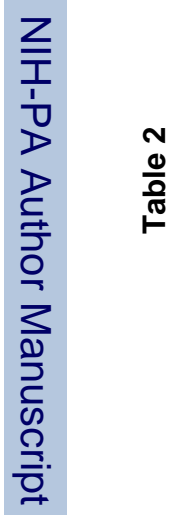

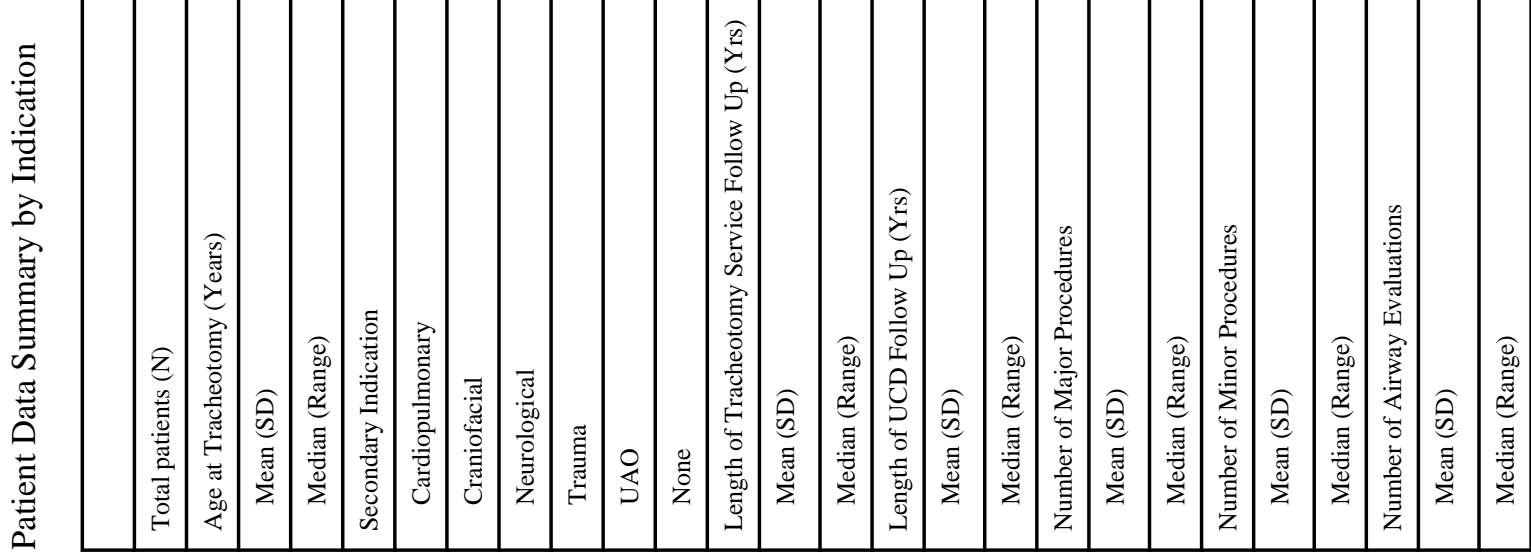



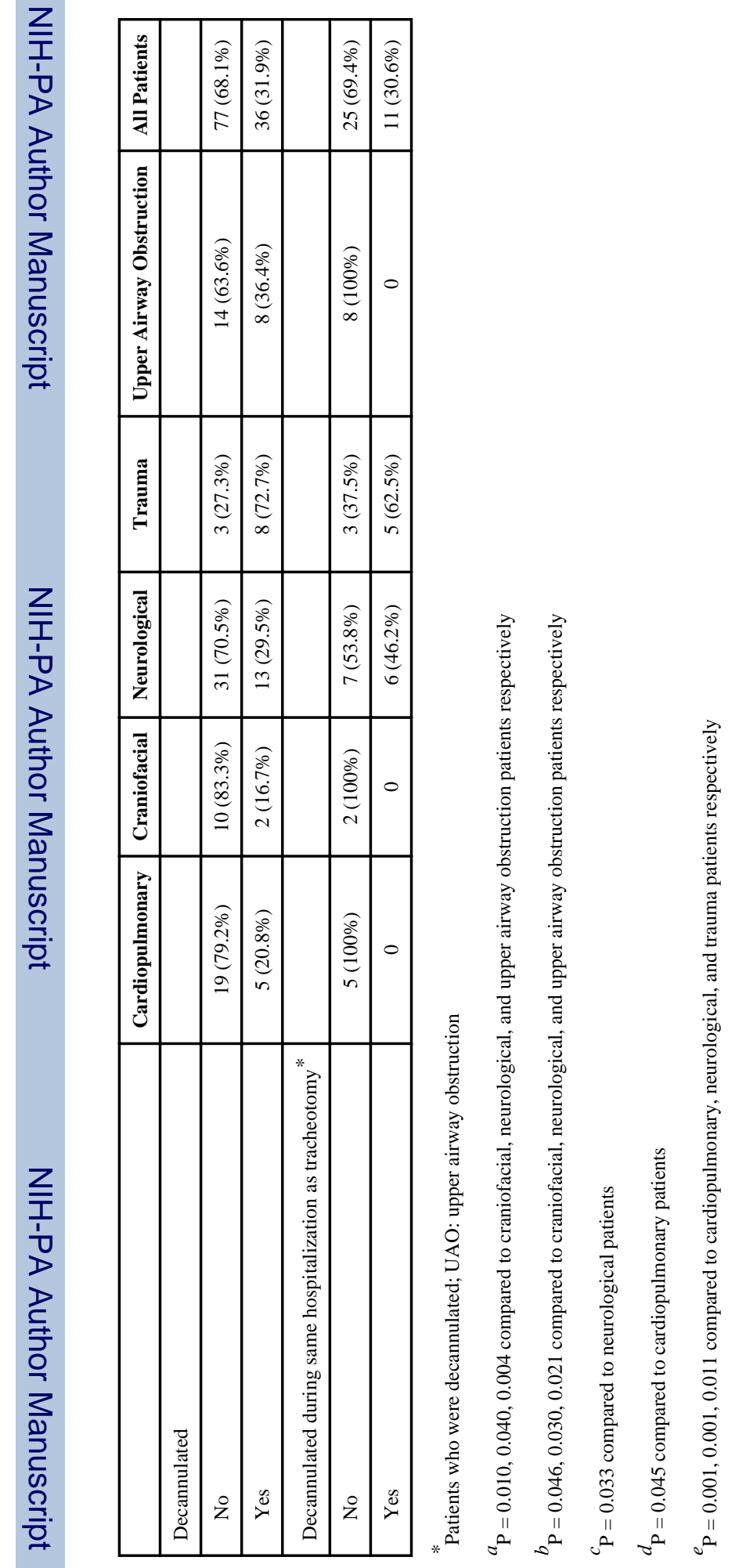


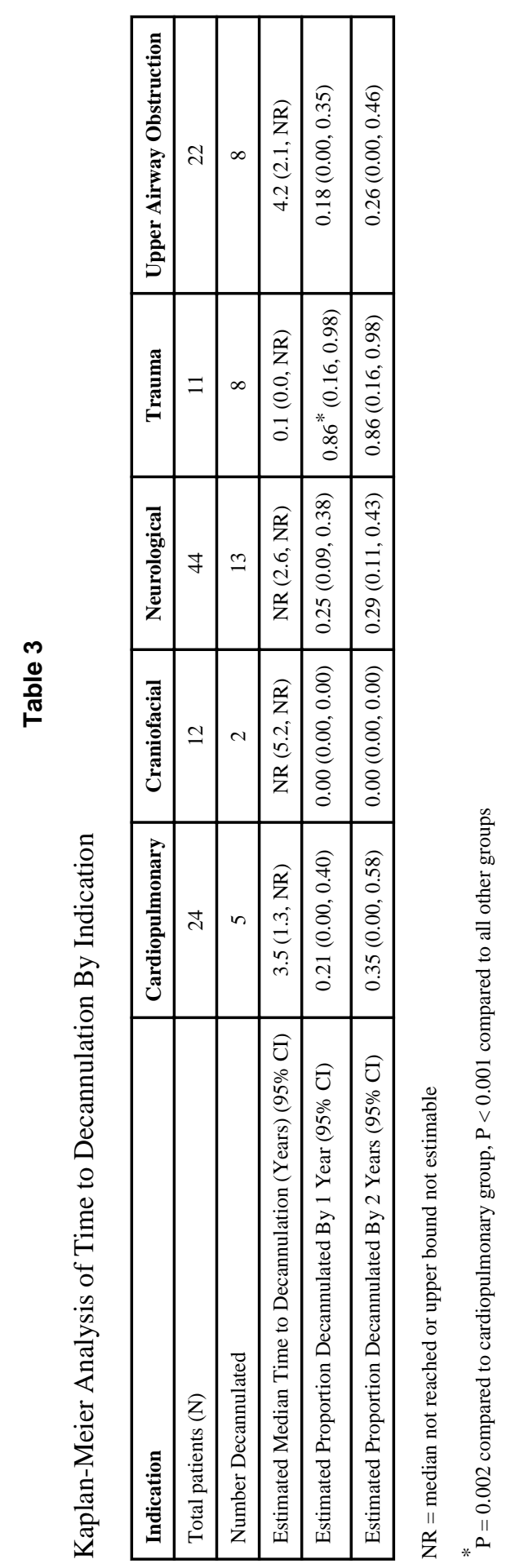


